\title{
Epidemiology of non-steroidal anti- inflammatory drugs consumption in Spain. The MCC-Spain study
}

Inés Gómez-Acebo ${ }^{1,2^{*}}$, Trinidad Dierssen-Sotos ${ }^{1,2}$, María de Pedro ${ }^{2,3}$, Beatriz Pérez-Gómez ${ }^{1,4,5}$, Gemma Castaño-Vinyals ${ }^{1,6,7,8}$, Tania Fernández-Villa9 ${ }^{9}$, Camilo Palazuelos-Calderón², Pilar Amiano ${ }^{1,10}$, Jaione Etxeberria ${ }^{1,11}$, Yolanda Benavente ${ }^{1,12}$, Guillermo Fernández-Tardón 1,13, Inmaculada Salcedo-Bellido 1,14, Rocío Capelo ${ }^{15}$, Rosana Peiró ${ }^{1,16}$, Rafael Marcos-Gragera ${ }^{1,17}$, José M. Huerta ${ }^{1,18}$, Adonina Tardón ${ }^{1,13}$, Aurelio Barricarte 1,19,20, Jone-Miren Altzibar ${ }^{1,21}$, Jessica Alonso-Molero², Verónica Dávila-Batista9 Nuria Aragonés ${ }^{1,4,5,22}$, Marina Pollán ${ }^{1,4,5}$, Manolis Kogevinas ${ }^{1,6,7,8}$ and Javier Llorca ${ }^{1,2}$

\begin{abstract}
Background: Non-steroidal anti-inflammatory drugs (NSAIDs) are widely used despite their risk of gastrointestinal bleeding or cardiovascular events. We report the profile of people taking NSAIDs in Spain, and we include demographic factors, health-related behaviours and cardiovascular disease history.

Methods: Four thousand sixtyparticipants were selected using a pseudorandom number list from Family Practice lists in 12 Spanish provinces. They completed a face-to-face computerized interview on their NSAID consumption, demographic characteristics, body mass index, alcohol and tobacco consumption and medical history. In addition, participants completed a self-administered food-frequency and alcohol consumption questionnaire. Factors associated with ever and current NSAID consumption were identified by logistic regression.

Results: Women consumed more non-aspirin NSAIDs (38.8\% [36.7-41.0]) than men (22.3 [20.5-24.2]), but men consumed more aspirin (11.7\% [10.3-13.2]) than women (5.2\% [4.3-6.3]). Consumption of non-aspirin NSAIDs decrease with age from $44.2 \%$ (39.4-49.1) in younger than 45 to $21.1 \%(18.3-24.2)$ in older than 75 , but the age-pattern for aspirin usage was the opposite. Aspirin was reported by about 11\% patients, as being twice as used in men (11.7\%) than in women (5.2\%); its consumption increased with age from 1.7\% ( $<45$ years old) to 12.4\% ( $\geq 75$ years old). Aspirin was strongly associated with the presence of cardiovascular risk factors or established cardiovascular disease, reaching odds ratios of 15.2 (7.4-31.2) in women with acute coronary syndrome, 13.3 (6.2-28.3) in women with strokes and 11.1 (7.8-15.9) in men with acute coronary syndrome. Participants with cardiovascular risk factors or diseases consumed as much non-aspirin NSAID as participants without such conditions.

Conclusions: Non-aspirin NSAIDs were more consumed by women and aspirin by men. The age patterns of aspirin and non-aspirin NSAIDs were opposite: the higher the age, the lower the non-aspirin NSAIDs usage and the higher the aspirin consumption. People with cardiovascular risk factors or diseases consumed more aspirin, but they did not decrease their non-aspirin NSAIDs usage.
\end{abstract}

Keywords: Aspirin, Cardiovascular risk, Gastrointestinal bleeding, Non-steroidal anti-inflammatory drugs, Propionates

\footnotetext{
* Correspondence: ines.gomez@unican.es

${ }^{1}$ CIBER Epidemiología y Salud Pública, Madrid, Spain

${ }^{2}$ Facultad de Medicina, Universidad de Cantabria - IDIVAL, Avda Herrera Oria

s/n, 39011 Santander, Spain

Full list of author information is available at the end of the article
}

(c) The Author(s). 2018 Open Access This article is distributed under the terms of the Creative Commons Attribution 4.0 International License (http://creativecommons.org/licenses/by/4.0/), which permits unrestricted use, distribution, and reproduction in any medium, provided you give appropriate credit to the original author(s) and the source, provide a link to the Creative Commons license, and indicate if changes were made. The Creative Commons Public Domain Dedication waiver (http://creativecommons.org/publicdomain/zero/1.0/) applies to the data made available in this article, unless otherwise stated. 


\section{Background}

Non-steroidal anti-inflammatory drugs (NSAIDs) are one of the most used therapeutic groups of agents; they can be obtained over-the-counter in many countries, and they are used for a wide variety of indications, including short-term and long-term treatment of pain, traumatisms, inflammatory diseases such as arthritis, rheumatoid arthritis and many others. On the other hand, NSAIDs can be responsible for several well-known side effects, comprising upper gastrointestinal bleeding [1] and cardiovascular disease [2]. While gastrointestinal haemorrhage would partially be prevented by adding proton-pump inhibitors to NSAIDs $[3,4]$, there is still some controversy regarding the differences in cardiovascular risk among the NSAID family [5].

Although the consumption of NSAIDs in Spain has decreased from 43.1 in the year 2013 to 37.9 in 2016, and this decrease in consumption was observed in all subgroups [6]; some studies have shown a trend towards increasing NSAID usage in developed countries [7, 8]. Little is known, however, about the medical characteristics of the consumers. In this way, medical records would be insufficient to establish patient profile, as a relevant NSAID amount is traded over-the-counter. Demographic characteristics are associated with different adverse effect risks. For instance, gastrointestinal haemorrhage is more frequent in elder people taking NSAIDs than in youngsters [1], and similar considerations could are responsible for cardiovascular effects [2]. Moreover, some health-related behaviours, such as alcohol [9] or -we speculate- tobacco usage, if associated with NSAID consumption, could potentiate their risk of cardiovascular episodes or gastrointestinal bleeding. Thus, Chi et al. observed that the proportions of patients with concomitant antiplatelet drugs, $\mathrm{H}$ pylori infection and status of smoking were also considerably higher in GI (gastrointestinal) bleeding group compared to non-GI bleeding group GI bleeding group associated with NSAIDs drugs [10] and Sostres et al. also observed that a higher risk of upper GI bleeding was associated with current or past smoking habit and previous history of peptic ulcer [11].

The aim of this study is to describe demographic characteristics related to NSAID consumption in the adult population in Spain, as well as health-related behaviors and cardiovascular risk factors. In order to do this, we analysed the control sample (about 4000 subjects) in the MCC-Spain project, a multi-centre case-control study carried out in Spain.

\section{Methods}

MCC-Spain is a case-control study on cancer carried out in 12 Spanish provinces: Asturias, Barcelona, Cantabria, Girona, Granada, Gipuzkoa, Huelva, León, Madrid, Murcia, Navarra, and Valencia [12]. More than 10,000 patients were recruited from 2009 to 2012, including cases of colorectal, breast, prostate or gastric cancer, and chronic lymphoid leukaemia, and 4062 controls frequency matched by age, sex and area of recruitment. In this article, only the control sample will be analysed, so all references to patients, subjects or participants from here on refer to the control sample. The study design, sample size and data gathering were planned for the case-control study.

Participants were recruited using computer-generated pseudorandom numbers from the list of patients assigned to general practice clinics. Selected people were contacted by phone; if contact with the selected person was not possible after a minimum of five tries at different times of the day, or if he/she refused to participate, the following person on the list was approached. In the Spanish Health System, every inhabitant is assigned to a general practice clinic irrespective of whether he or she does attend to that clinic; therefore, selecting by random from those lists did not introduce a bias towards sick people. Participants who agreed to partake in the study signed an informed consent before the face-to-face interview, and the protocol of MCC-Spain was approved by the local Ethics Committees of participating institutions (Comité Ético de Investigación Clínica (CEIC) del Instituto Municipal de Asistencia Sanitaria de Barcelona; CEIC del Hospital Universitario de Bellvitge; CEIC de Navarra; CEIC del Hospital Universitario La Paz; CEIC del Hospital Universitario Ramón y Cajal; CEIC de Cantabria; CEIC de Gipuzkoa; CEIC de Girona; Comité de Ética de la Investigación de la Provincia de Huelva; CEIC de León; Comité Ético de Investigación del Principado de Asturias), in conformity to the principles of the Declaration of Helsinki. The database was registered in the Spanish Agency for Data Protection (no. 2102672171).

A structured computerized epidemiological questionnaire was administered by trained personnel in a face-to-face interview to get information on demographics, anthropometrics, family history of cancer, history of diseases, drug consumption, occupational history, health behaviours, and reproductive factors [13]. Usage of NSAIDs was specifically asked about using a detailed questionnaire including the specific NSAID, age at beginning, age when end duration of consumption and current consumption; a participant was considered as having taken a specific NSAID if she/he reported to have taken at least 30 doses. We carried out separated analyses for ever and current consumers of NSAIDs; current consumption could be consequence of recent conditions, while ever consumption better represents cumulative exposure to NSAIDs but it could be more prone to recall bias.

On the other hand, participants were provided with a semi-quantitative Food Frequency Questionnaire (FFQ) previously validated in the Spanish population [14], which included questions on alcohol consumption both currently and at 30-40 years old [15]. The FFQ was self-administered and returned by mail or filled out face to face within a period not exceeding 15 days after the interview [13]. Only 
3509 participants answered this questionnaire. Alcohol consumption was asked for every type of beverage; for instance, we asked "How often do you drink one glass of red wine?", giving the options: never or less than 1 time per month / 1-3 per month / 1-2 per week / 3-4 per week / 5-6 per week / 1 per day / 2-3 per day / 2-3 per day / 4 or more per day. Then we assumed a glass of wine being $100 \mathrm{cL}$, containing 12\% alcohol. Average alcohol drinking was classified in abstainer (less than one drink per month), category I (0-19.9 g /day for women and 0-39.9 g/day for men), category II (20-39.9 g/day for women, 40-59.9 g/ day for men) and category III ( $\geq 40 \mathrm{~g} /$ day for women, $\geq 60 \mathrm{~g} /$ day for men), according to the comparative risk assessment module of the Global Burden of Disease [16]. For instance, it would be necessary to take 4 glasses of wine or 2 cups of whisky to reach $40 \mathrm{~g}$ of alcohol. NSAIDs were classified according to the Anatomical and Therapeutic Classification of Drugs (ATC) in aspirin (ATC code N02BA01), butilpirazone (M01aa), acetic derivatives (M01ab), oxicams (M01 ac), propionates (M01ae), coxibs (M01ah) and others (M01ax).

A separated analysis was carried out to ascertain NSAID consumption in people with cardiovascular diseases or risk factors, as current clinical guidelines point out the increase of cardiovascular risk associated with non-aspirin NSAIDs.

Proportions and their 95\% confidence intervals (CI) were estimated assuming a binomial distribution. Variables associated with NSAID consumption were identified by binomial logistic regression; its results are displayed as odds ratios (OR) with 95\% CI. The statistical package Stata 14/SE was used for the analysis (Stata Corp, College Station, Tx, US).

\section{Results}

Characteristics of the 4060 controls included in this analysis are reported in Table 1 . They were 2023 women and 2037 men, with ages ranging $22-85 ; 49.4 \%$ had reached secondary or university education. $61.8 \%$ subjects were overweight or obese, $19.2 \%$ were current smokers and, when being $30-40$ years old, $16 \%$ had an average alcohol consumption higher than $20 \mathrm{~g} /$ day in women and $40 \mathrm{~g} /$ day in men. About one participant in four suffered arthritis, $10 \%$ had chronic cephalalgia and $5.6 \%$, gout. Arthritis and chronic cephalalgia were more frequent in women.

Figure 1 and Additional file 1 reported the ever NSAID consumption frequency by age and sex. About $30 \%$ subjects reported non-aspirin NSAID consumption, showing a step-down trend with age, from $44.2 \%$ in subjects under 45 to $21.1 \%$ in patients over 75 . Women consumed NSAID at higher rates than men (38.8\% vs. $22.3 \%)$; this gender pattern was consistent among all ages. The most consumed NSAID group was propionates (M01ae) (29.2\%) with, again, a consistent age and sex pattern: higher consumption in women and in youngsters. Aspirin was reported by about $11 \%$ patients, being twice as used in men (11.7\%) than in women (5.2\%); its consumption increased with age from $1.7 \%$ ( $<45$ years old) to $12.4 \%$ ( $\geq 75$ years old). Acetate derivatives (M01ab) -the third most consumed group- was reported in similar percentages by both sexes, without a neat trend with age. Consumption of the remaining groups was scarce (butylpyrazolidines (M01aa): 0.03\%, oxicam (M01 ac): 0.6\%, coxib (M01ah): 0.6\%, others (M01ax): 2.19\%) and we did not carry out additional analyses on them. Consumption of NSAIDs at the time of recruitment is reported in Fig. 2 and Additional file 2. Women used non-aspirin NSAIDs twice as much as men $(20.7 \%$ for women vs. $9.0 \%$ for men); again, this pattern was consistent across age groups, with non-aspirin NSAID consumption decreasing from $22.1 \%$ in younger than 45 years to $10.4 \%$ in older than 75. Aspirin, however, was more consumed by men $(9.0 \%)$ than for women $(2.9 \%)$ and its rates increased with age in both men and women. About two thirds of the non-aspirin NSAID consumption was due to propionates; in men, the consumption declined from $9.4 \%$ in younger than 45 years old to $2.7 \%$ in men older than 75 ; in women, the decrease was from $20.7 \%$ ( $<45$ years) to $9.4 \%$ ( $>75$ years).

Consumption by province (Additional file 3) was heterogeneous ranging from $13.4 \%$ (in Granada) to $45.1 \%$ in Girona) for non-aspirin NSAIDs.

The most frequently ever used specific NSAIDs were ibuprofen (20.4\%), aspirin (11\%) and diclofenac (6.4\%). Only three other drugs were used by more than $1 \%$ of people: aciclofenac (1.5\%), naproxen $(1.4 \%)$ and chondroitin sulphate (1.3\%). Regarding current consumption, only ibuprofen (9.2\%), aspirin (5.9\%), diclofenac (2.0\%) and chondroitin sulphate $(1.0 \%)$ reached the $1 \%$ cut off (Additional file 4).

\section{Factors related to NSAIDs ever consumption}

Tables 2, 3, 4 and 5 report the factors associated with consumption of the main NSAID groups in men and women, according to the multivariate logistic regression analysis. Non-aspirin NSAID were less consumed in men as age increased (reaching OR $=0.26$ in men older than 75 compared to men between 45 and 54 years old) and as education level goes up, being about twice as frequent in men with university level than in men without primary studies; no association was found among non-aspirin NSAID consumption in men and body mass index (BMI)or ethanol consumption. However, men who currently use NSAIDs that are not aspirin were half less likely $(\mathrm{OR}=0.49)$ to be current smokers than non-smokers. If we consider chronic disease involving pain, men who had arthritis multiplied by almost 3 the probability of taking non-aspirin NSAID and doubled its use in other chronic disease involving pain that were not arthritis gout or chronic cephalalgia (Table 2). Similar 
Table 1 Descriptive analysis of sociodemographic variables

\begin{tabular}{|c|c|c|c|c|}
\hline \multirow[t]{2}{*}{ Variable } & \multirow[t]{2}{*}{ Category } & \multirow{2}{*}{$\begin{array}{l}\text { Total } \\
\text { Frequency (\%) } \\
\text { or MEAN } \pm \text { SD }\end{array}$} & \multirow{2}{*}{$\begin{array}{l}\text { Women } \\
(N=2023,49.8 \%) \\
\text { Frequency }(\%) \\
\text { or MEAN } \pm \text { SD }\end{array}$} & \multirow{2}{*}{$\begin{array}{l}\text { Men } \\
(N=2037,50.2 \%) \\
\text { Frequency }(\%) \\
\text { or MEAN } \pm \text { SD }\end{array}$} \\
\hline & & & & \\
\hline Age (years) & Continuous & $62.9 \pm 12.1$ & $59.3 \pm 13.3$ & $66.5 \pm 9.6$ \\
\hline \multirow[t]{5}{*}{ Age (years) } & $22-44$ & $421(10.4)$ & $357(17.7)$ & $64(3.1)$ \\
\hline & $45-54$ & $579(14.3)$ & $428(21.2)$ & $151(7.4)$ \\
\hline & $55-64$ & $1013(25.0)$ & $444(22.0)$ & $569(27.9)$ \\
\hline & $65-74$ & $1278(31.5)$ & $476(23.5)$ & $803(39.4)$ \\
\hline & $75-85$ & 769 (18.9) & $318(15.7)$ & $452(22.2)$ \\
\hline \multirow[t]{4}{*}{ Education level } & Lower than primary & $755(18.6)$ & $372(18.4)$ & $383(18.8)$ \\
\hline & Primary education & $1301(32.0)$ & $616(30.5)$ & $686(33.6)$ \\
\hline & Secondary education & $1172(28.9)$ & $616(30.5)$ & $556(27.3)$ \\
\hline & University & $832(20.5)$ & $419(20.7)$ & $414(20.3)$ \\
\hline \multirow[t]{4}{*}{ Body mass index $\left(\mathrm{kg} / \mathrm{m}^{2}\right)$} & $<18.5$ & $54(1.3)$ & $45(2.29$ & $9(0.4)$ \\
\hline & $18.5-24.9$ & $1498(36.9)$ & $946(46.8)$ & $553(27.1)$ \\
\hline & 25-29.9 (overweight) & $1661(40.9)$ & 640 (31.6) & $1022(50.1)$ \\
\hline & $\geq 30$ (obesity) & $847(20.9)$ & $392(19.4)$ & $455(22.3)$ \\
\hline \multirow[t]{3}{*}{ Smoking } & No smoker & $1812(44.6)$ & $1236(61.1)$ & $576(28.3)$ \\
\hline & Current smoker & $780(19.2)$ & $375(18.5)$ & $405(19.9)$ \\
\hline & Former smoker & $1468(36.2)$ & $1057(51.8)$ & $411(20.4)$ \\
\hline \multirow{4}{*}{$\begin{array}{l}\text { Alcohol consumption at recruitment } \\
\text { (g/day) }\end{array}$} & Abstainers & $939(26.8)$ & $637(37.0)$ & $302(16.9)$ \\
\hline & 0-19.9 (women), 0-39.9 (men) & $2295(65.4)$ & $999(58.1)$ & $1296(72.4)$ \\
\hline & 20-39.9 (women), 40-59.9 (men) & $203(5.8)$ & $74(4.3)$ & $129(7.2)$ \\
\hline & $\geq 40$ (women), $\geq 60$ (men) & $72(2.0)$ & $9(0.5)$ & $63(3.5)$ \\
\hline \multirow{4}{*}{$\begin{array}{l}\text { Alcohol consumption when } \\
30-40 \text { years old ( } \mathrm{g} / \text { day) }\end{array}$} & 0 & $903(25.7)$ & $657(38.3)$ & $246(13.7)$ \\
\hline & 0-19.9 (women), 0-39.9 (men) & $2047(58.3)$ & $951(55.3)$ & $1096(61.2)$ \\
\hline & 20-39.9 (women), 40-59.9 (men) & $300(8.6)$ & $86(5.0)$ & $214(12.0)$ \\
\hline & $\geq 40$ (women), $\geq 60$ (men) & $259(7.4)$ & $24(1.4)$ & $235(13.1)$ \\
\hline \multirow[t]{3}{*}{ Number of births(women) } & 0 & & $375(18.5)$ & \\
\hline & $1-2$ & & $1085(53.6)$ & \\
\hline & $>2$ & & $563(27.9)$ & \\
\hline \multirow[t]{2}{*}{ Menopausal status (women) } & Premenopausal & & $650(32.1)$ & \\
\hline & Postmenopausal & & $1373(67.9)$ & \\
\hline \multirow[t]{4}{*}{ Chronic disease involving pain } & Arthritis & $1005(24.9)$ & $626(31.1)$ & $379(18.7)$ \\
\hline & Gout & $225(5.6)$ & $24(1.2)$ & $201(9.9)$ \\
\hline & Chronic cephalalgia & $414(10.2)$ & $307(15.3)$ & $107(5.3)$ \\
\hline & Arthritis, gout or chronic cephalalgia & $1425(35.1)$ & $816(40.3)$ & 609 (29.9) \\
\hline \multirow{6}{*}{$\begin{array}{l}\text { Cardiovascular disease or risk } \\
\text { factor }\end{array}$} & Diabetes mellitus & $594(14.7)$ & $191(9.5)$ & $403(19.8)$ \\
\hline & Hypertension & $1513(37.4)$ & $587(29.2)$ & $926(45.6)$ \\
\hline & Hypercholesterolemia & $1344(33.3)$ & $580(28.9)$ & $764(37.7)$ \\
\hline & Acute coronary syndrome & $312(7.7)$ & $58(2.9)$ & $254(12.5)$ \\
\hline & Stroke & $151(3.7)$ & $53(2.6)$ & $94(4.8)$ \\
\hline & Other circulatory diseases & $628(15.5)$ & $330(16.4)$ & $298(14.7)$ \\
\hline
\end{tabular}



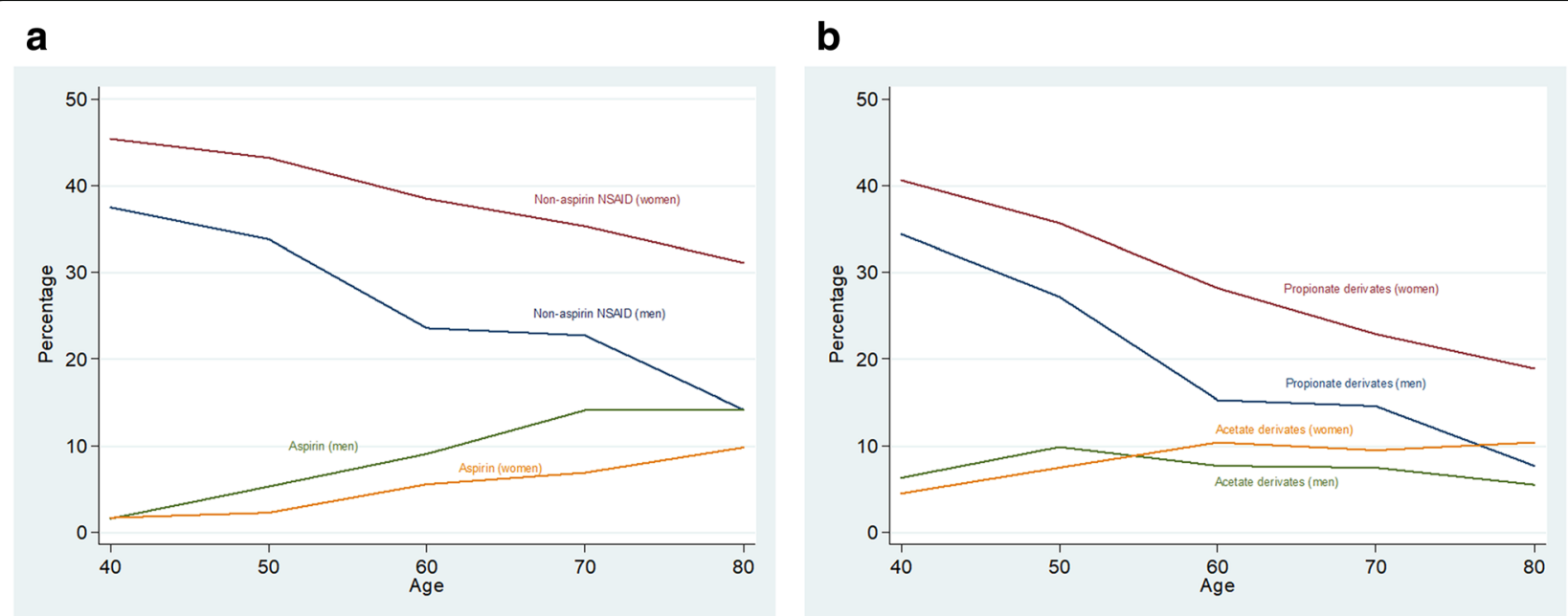

Fig. 1 Anytime consumption of NSAID by age and sex. 1a: Aspirin and non-aspirin NSAID. 1b: Propionates and acetate derivates

patterns were found in women, although both age and education levels showed less apparent trends. Number of births and menopausal status were not associated with non-aspirin NSAIDs consumption. Patients with chronic conditions involving pain (arthritis, gout or chronic cephalalgia) consumed non-aspirin NSAIDs four times as much as participants without such conditions. In contrast than men, women who once used NSAIDs without aspirin were approximately $38 \%$ more likely to be current smokers than nonsmokers and use in chronic cephalalgia (OR 2.54 for ever or OR 1.66 for current use).

As propionate derivatives were the more frequently consumed NSAID group, its related factors (Table 3) resembled those of non-aspirin NSAIDs: the higher the age, the lower the propionate derivative consumption, and for men, the higher the education level, the higher their propionate derivative consumption. People with conditions with chronic pain (arthritis, chronic cephalalgia or gout) used propionate derivates twice or three times more frequently than people without such conditions. Multivariate results in aspirin consumptions are displayed in Table 4. Men increased their aspirin consumption with age, being about three times higher in men older than 65 , and smoking habit, with current or former smokers having $60 \%$ higher aspirin consumption than non-smoking men. In women, however, only age and number of births $(\mathrm{OR}=1.24$ for each birth) increased aspirin consumption.

Factors associated with acetate derivates are analyzed in Table 5. Chronic diseases involving pain multiplied by 2.7 (women) and 3.5 (men) the odds of having used acetate derivates. Apart from this factor, in men, only the a

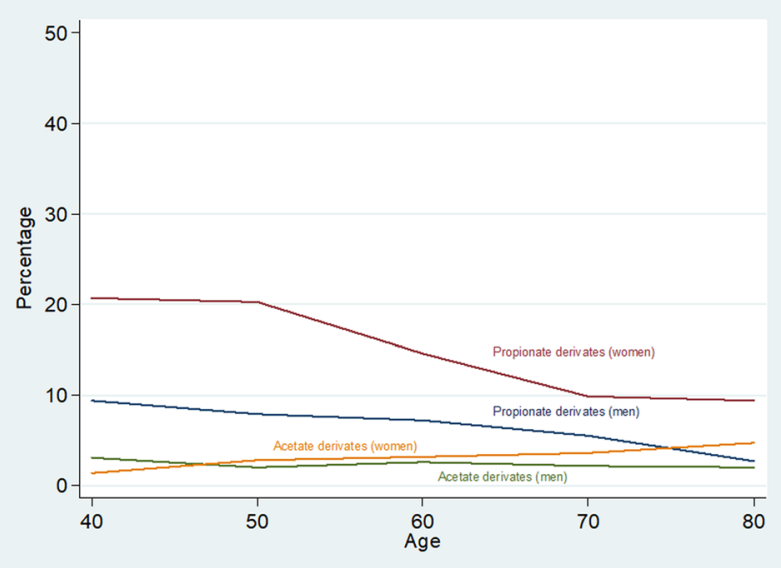

b

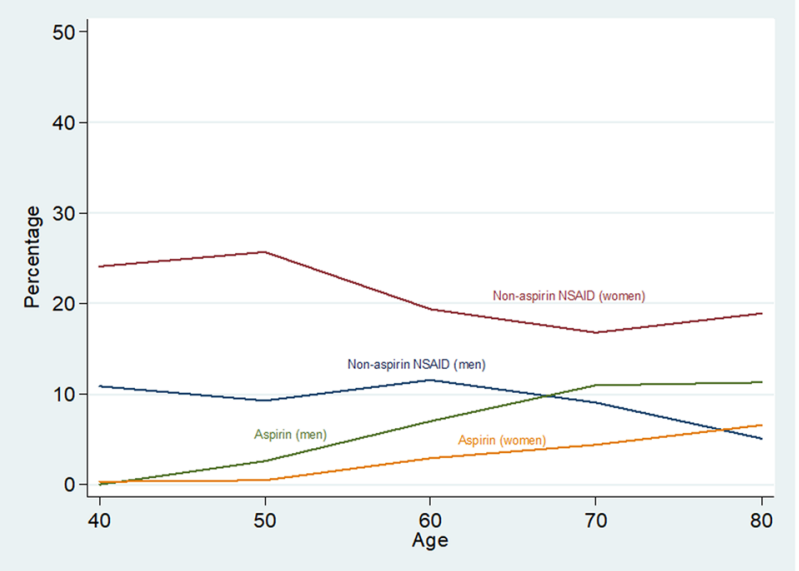

Fig. 2 Current consumption of NSAID by age and sex. 1a: Aspirin and non-aspirin NSAID. 1b: Propionates and acetate derivates 
Table 2 Factors associated with non-aspirin NSAID consumption: odds ratios and 95\% confidence intervals adjusted for the remaining factors in the table and province of recruitment

\begin{tabular}{|c|c|c|c|c|c|}
\hline \multirow[t]{2}{*}{ Variable } & \multirow[t]{2}{*}{ Category } & \multicolumn{2}{|c|}{ Ever consumption } & \multicolumn{2}{|c|}{ Current consumption } \\
\hline & & Women & Men & Women & Men \\
\hline \multirow[t]{5}{*}{ Age } & $<45$ & $1.50(1.04-2.15)$ & $1.23(0.62-2.44)$ & $1.17(0.78-1.75)$ & $1.57(0.56-4.42)$ \\
\hline & $45-54$ & 1 (ref) & 1 (ref) & 1 (ref) & 1 (ref) \\
\hline & $55-64$ & $0.58(0.40-0.85)$ & $0.53(0.34-0.83)$ & $0.62(0.41-0.96)$ & $1.27(0.63-2.55)$ \\
\hline & $65-74$ & $0.39(0.26-0.59)$ & $0.45(0.29-0.70)$ & $0.39(0.24-0.62)$ & $0.76(0.38-1.54)$ \\
\hline & $\geq 75$ & $0.39(0.24-0.62)$ & $0.26(0.16-0.43)$ & $0.46(0.27(0.78)$ & $0.37(0.17-0.84)$ \\
\hline \multirow[t]{4}{*}{ BMl } & $<18.5$ & $1.06(0.53-2.15)$ & $1.75(0.23-13.6)$ & $0.39(0.14-1.07)$ & $3.50(0.28-44.3)$ \\
\hline & $18.5-24.9$ & 1 (ref) & 1 (ref) & 1 (ref) & 1 (ref) \\
\hline & $25-29.9$ & $0.94(0.73-1.21)$ & $0.99(0.74-1.32)$ & $0.94(0.70-1.27)$ & $0.90(0.60-1.37)$ \\
\hline & $\geq 30$ & $1.38(1.02-1.88)$ & $0.85(0.59-1.22)$ & $1.30(0.92-1.84)$ & $1.06(0.65-1.74)$ \\
\hline \multirow[t]{4}{*}{ Education } & Less than primary & 1 (ref) & 1 (ref) & 1 (ref) & 1 (ref) \\
\hline & Primary education & $1.31(0.92-1.85)$ & $1.56(1.04-2.33)$ & $0.91(0.61-1.36)$ & $1.11(0.63-1.98)$ \\
\hline & Secondary education & $1.21(0.83-1.76)$ & $2.10(1.39-3.16)$ & $0.90(0.58-1.39)$ & $1.30(0.72-2.33)$ \\
\hline & University & $1.70(1.12-2.58)$ & $2.55(1.65-3.95)$ & $1.29(0.80-2.07)$ & $2.12(1.15-3.89)$ \\
\hline \multicolumn{2}{|c|}{ No. of births (by each birth) } & $1.04(0.97-1.12)$ & & $1.05(0.96-1.14)$ & \\
\hline \multicolumn{2}{|c|}{ Premenopause (ref:: postmenopause) } & $0.86(0.61-1.22)$ & & $1.15(0.77-1.70)$ & \\
\hline \multirow[t]{3}{*}{ Smoking } & No smoker & 1 (ref) & 1 (ref) & 1 (ref) & 1 (ref) \\
\hline & Former smoker & $1.20(0.90-1.59)$ & $1.03(0.77-1.36)$ & $1.00(0.72-1.39)$ & $0.98(0.66-1.45)$ \\
\hline & Current smoker & $1.38(1.02-1.88)$ & $0.76(0.52-1.09)$ & $1.07(0.76-1.53)$ & $0.49(0.27-0.88)$ \\
\hline \multicolumn{6}{|c|}{ Alcohol consumption at recruitment (g/day) } \\
\hline \multicolumn{2}{|c|}{ Abstainers } & 1 (ref) & 1 (ref) & 1 (ref) & 1 (ref) \\
\hline \multicolumn{2}{|c|}{ 0-39.9 (men), 0-19.9 (women) } & $1.03(0.76-1.39)$ & $1.26(0.85-1.88)$ & $0.78(0.55-1.11)$ & $1.06(0.58-1.92)$ \\
\hline \multicolumn{2}{|c|}{ 40-59.9 (men), 20-39.9 (women } & $1.11(0.57-2.14)$ & $1.60(0.89-2.89)$ & $0.58(0.25-1.30)$ & $1.16(0.49-2.79)$ \\
\hline \multicolumn{2}{|c|}{$\geq 60$ g/day (men), $\geq 40$ g/day (women) } & $1.22(0.26-5.68)$ & $1.95(0.94-4.02)$ & $3.03(0.56-16.6)$ & $2.69(0.97-7.44)$ \\
\hline \multicolumn{6}{|c|}{ Alcohol when $30-40$ years old } \\
\hline \multicolumn{2}{|c|}{ Abstainers } & 1 (ref) & 1 (ref) & 1 (ref) & 1 (ref) \\
\hline \multicolumn{2}{|c|}{ 0-39.9 (men), 0-19.9 (women) } & $1.01(0.75-1.36)$ & $0.94(0.62-1.42)$ & $1.30(0.92-1.84)$ & $1.88(0.96-3.68)$ \\
\hline \multicolumn{2}{|c|}{ 40-59.9 (men), 20-39.9 (women } & $1.28(0.69-2.36)$ & $1.08(0.64-1.82)$ & $1.93(0.95-3.93)$ & $2.11(0.94-4.72)$ \\
\hline \multicolumn{2}{|c|}{$\geq 60 \mathrm{~g} /$ day (men), $\geq 40 \mathrm{~g} /$ day (women) } & $0.97(0.37-2.58)$ & $0.81(0.48-1.36)$ & $1.42(0.45-4.47)$ & $0.78(0.32-1.87)$ \\
\hline \multicolumn{6}{|c|}{ Chronic disease involving pain } \\
\hline \multicolumn{2}{|c|}{ Yes (ref: No) } & $4.31(3.37-5.50)$ & $3.60(2.78-4.67)$ & $3.77(2.85-4.99)$ & $4.59(3.18-6.61)$ \\
\hline
\end{tabular}

educational level reached a positive significant relationship with consumption; men with secondary or university education reported about twice the acetate derivative consumption than men with lower educational levels. No other factor can be identified as associated with acetate derivative consumption in women. BMI displayed a non-significant association in both men and women.

\section{Factors related to current consumption}

Non-aspirin NSAIDs consumption (Table 2) stepped down with age in both women $(\mathrm{OR}=1.17,1,0.62,0.39$ and 0.46 for the ordered age groups) and men $(\mathrm{OR}=1.57,1,1.27$, 0.76 and 0.37). Men with university level education consumed twice as much as men with less-than-primary level; a similar result was not found in women. Consumption of non-aspirin NSAIDs was halved in current smoking men. Regarding alcohol use, only participants in the highest category of current consumption (i.e.: $\geq 60 \mathrm{~g} /$ day for men and $\geq 40 \mathrm{~g} /$ day for women) reported higher non-aspirin NSAID used, although estimates were unstable for women due to the small number of women in this category. Suffering arthritis, gout or chronic cephalalgia multiplies the probability of using non-aspirin NSAIDs by about 4 .

Propionates consumption (Table 3) in men went down with age -reaching $\mathrm{OR}=0.19$ in men older than 75 - and in current smokers $(\mathrm{OR}=0.55)$. Suffering chronic pain 
Table 3 Factors associated with propionate derivate consumption: odds ratios and 95\% confidence intervals adjusted for the remaining factors in the table and province of recruitment

\begin{tabular}{|c|c|c|c|c|c|}
\hline \multirow[t]{2}{*}{ Variable } & \multirow[t]{2}{*}{ Category } & \multicolumn{2}{|c|}{ Ever consumption } & \multicolumn{2}{|c|}{ Current consumption } \\
\hline & & Women & Men & Women & Men \\
\hline \multirow[t]{5}{*}{ Age } & $<45$ & $1.47(1.02-2.12)$ & $1.48(0.73-3.00)$ & $1.17(0.76-1.79)$ & $1.33(0.45-3.94)$ \\
\hline & $45-54$ & 1 (ref) & 1 (ref) & 1 (ref) & 1 (ref) \\
\hline & $55-64$ & $0.54(0.36-0.80)$ & $0.45(0.28-0.73)$ & $0.72(0.45-1.16)$ & $0.85(0.41-1.76)$ \\
\hline & $65-74$ & $0.32(0.21-0.49)$ & $0.40(0.25-0.65)$ & $0.36(0.21-0.62)$ & $0.57(0.27-1.19)$ \\
\hline & $\geq 75$ & $0.30(0.18-0.51)$ & $0.20(0.11-0.35)$ & $0.38(0.20-0.71)$ & $0.19(0.07-0.50)$ \\
\hline \multirow[t]{4}{*}{ BMl } & $<18.5$ & $1.46(0.72-2.98)$ & $0.98(0.10-10.1)$ & $0.33(0.09-1.13)$ & - \\
\hline & $18.5-24.9$ & 1 (ref) & 1 (ref) & 1 (ref) & 1 (ref) \\
\hline & $25-29.9$ & $0.81(0.61-1.07)$ & $0.98(0.71-1.36)$ & $0.94(0.67-1.31)$ & $0.98(0.60-1.62)$ \\
\hline & $\geq 30$ & $1.38(1.00-1.90)$ & $0.81(0.54-1.23)$ & $1.35(0.92-1.98)$ & $1.33(0.75-2.39)$ \\
\hline \multirow[t]{4}{*}{ Education } & Less than primary & 1 (ref) & 1 (ref) & 1 (ref) & 1 (ref) \\
\hline & Primary education & $1.30(0.89-1.90)$ & $1.83(1.13-2.96)$ & $0.91(0.57-1.45)$ & $1.27(0.63-2.56)$ \\
\hline & Secondary education & $1.15(0.76-1.74)$ & $2.17(1.33-3.55)$ & $0.82(0.49-1.35)$ & $1.27(0.63-2.56)$ \\
\hline & University & $1.42(0.90-2.23)$ & $2.57(1.53-4.33)$ & $1.00(0.58-1.72)$ & $2.00(0.95-4.24)$ \\
\hline No. of births & (by each birth) & $1.08(0.99-1.17)$ & & $1.01(0.91-1.12)$ & - \\
\hline \multicolumn{2}{|c|}{ Premenopause (ref:: postmenopause) } & $1.16(0.80-1.67)$ & & $1.57(1.02-2.43)$ & - \\
\hline \multirow[t]{3}{*}{ Smoking } & No smoker & 1 (ref) & 1 (ref) & 1 (ref) & 1 (ref) \\
\hline & Former smoker & $1.09(0.81-1.48)$ & $1.05(0.76-1.46)$ & $1.01(0.70-1.46)$ & $0.95(0.60-1.51)$ \\
\hline & Current smoker & $1.13(0.82-1.56)$ & $0.74(0.48-1.14)$ & $1.10(0.75-1.62)$ & $0.55(0.28-1.08)$ \\
\hline \multicolumn{6}{|c|}{ Alcohol consumption at recruitment (g/day) } \\
\hline \multicolumn{2}{|l|}{ Abstainers } & 1 (ref) & 1 (ref) & 1 (ref) & 1 (ref) \\
\hline \multicolumn{2}{|c|}{ 0-39.9 (men), 0-19.9 (women) } & $1.22(0.89-1.68)$ & $1.06(0.67-1.66)$ & $0.75(0.51-1.10)$ & $0.91(0.47-1.77)$ \\
\hline \multicolumn{2}{|c|}{ 40-59.9 (men), 20-39.9 (women) } & $1.41(0.69-2.87)$ & $1.10(0.55-2.19)$ & $0.58(0.22-1.52)$ & $0.98(0.35-4.48)$ \\
\hline \multicolumn{2}{|c|}{$\geq 60 \mathrm{~g} /$ day (men), $\geq 40 \mathrm{~g} /$ day (women) } & $0.50(0.05-4.80)$ & $1.48(0.64-3.39)$ & $0.98(0.09-10.9)$ & $1.25(0.35-4.48)$ \\
\hline \multicolumn{6}{|c|}{ Alcohol when $30-40$ years old } \\
\hline \multicolumn{2}{|l|}{ Abstainers } & 1 (ref) & 1 (ref) & 1 (ref) & 1 (ref) \\
\hline \multicolumn{2}{|c|}{ 0-39.9 (men), 0-19.9 (women) } & $0.86(0.63-1.18)$ & $1.22(0.76-1.96)$ & $1.19(0.81-1.75)$ & $1.10(0.54-2.23)$ \\
\hline \multicolumn{2}{|c|}{ 40-59.9 (men), 20-39.9 (women) } & $0.79(0.40-1.54)$ & $1.15(0.63-2.12)$ & $1.29(0.55-3.01)$ & $1.26(0.52-3.06)$ \\
\hline \multicolumn{2}{|c|}{$\geq 60 \mathrm{~g} /$ day (men), $\geq 40 \mathrm{~g} /$ day (women) } & $0.55(0.17-1.75)$ & $0.94(0.51-1.73)$ & $0.60(0.12-3.11)$ & $0.62(0.24-1.63)$ \\
\hline \multicolumn{6}{|c|}{ Chronic disease involving pain } \\
\hline \multicolumn{2}{|c|}{ Yes (ref: No) } & $3.08(2.37-3.99)$ & $2.22(1.65-2.98)$ & $2.58(1.89-3.52)$ & $2.48(1.61-3.82)$ \\
\hline
\end{tabular}

conditions $(\mathrm{OR}=2.48)$ and having reached university level $(\mathrm{OR}=2.00)$ were the only factors associated with higher propionate used. In women, higher ages were also associated with lower propionate consumption $(\mathrm{OR}=0.38$ in women $>75$ years) and the presence of chronic pain diseases, with higher consumption $(\mathrm{OR}=2.58)$; no other factor could be identified as associated with this NSAID group.

Higher age was a risk factor for using aspirin in both women and men (Table 4), with OR for the elder group reaching 6.8 (women) and 4.7 (men). The education level was negatively associated with aspirin consumption in women, but not in men, while smokers (both former and current) were related with higher aspirin use in men. Suffering from a chronic pain condition was not associated with aspirin consumption.

Apart from chronic pain diseases, which were associated with 3 or 4 times higher acetate derivate consumption (Table 5), only non-significant positive associations were found between this NSAID group and alcohol consumption (both sexes) and higher education level (men, but not women).

\section{NSAID consumption and cardiovascular disease or risk factors}

To further explore the risk profile of cardiovascular adverse events while taking NSAIDs, we analysed the 
Table 4 Factors associated with aspirin consumption: odds ratios and 95\% confidence intervals adjusted for the remaining factors in the table and province of recruitment

\begin{tabular}{|c|c|c|c|c|c|}
\hline \multirow[t]{2}{*}{ Variable } & \multirow[t]{2}{*}{ Category } & \multicolumn{2}{|c|}{ Ever consumption } & \multicolumn{2}{|c|}{ Current consumption } \\
\hline & & Women & Men & Women & Men \\
\hline \multirow[t]{5}{*}{ Age } & $<45$ & $0.62(0.20-1.96)$ & $0.25(0.03-2.06)$ & $0.76(0.06-9.39)$ & - \\
\hline & $45-54$ & 1 (ref) & 1 (ref) & 1 (ref) & 1 (ref) \\
\hline & $55-64$ & $2.03(0.81-5.13)$ & $1.54(0.70-3.40)$ & $2.99(0.57-15.7)$ & $2.35(0.81-6.84)$ \\
\hline & $65-74$ & $2.26(0.85-6.01)$ & $2.41(1.12-5.19)$ & $4.90(0.91-26.3)$ & $3.99(1.41-11.3)$ \\
\hline & $\geq 75$ & $3.59(1.26-10.2)$ & $3.09(1.39-6.87)$ & $6.83(1.21-38.7)$ & $4.74(1.62-13.9)$ \\
\hline \multirow[t]{4}{*}{ BMI } & $<18.5$ & $1.77(0.37-8.38)$ & - & $2.01(0.24-17.1)$ & - \\
\hline & $18.5-24.9$ & 1 (ref) & 1 (ref) & 1 (ref) & 1 (ref) \\
\hline & $25-29.9$ & $0.75(0.41-1.36)$ & $1.02(0.70-1.47)$ & $0.68(0.31-1.53)$ & $2.35(0.81-6.84)$ \\
\hline & $\geq 30$ & $1.73(0.98-3.07)$ & $1.32(0.85-2.04)$ & $1.42(0.66-3.05)$ & $1.45(0.89-2.38)$ \\
\hline \multirow[t]{4}{*}{ Education } & Less than primary & 1 (ref) & 1 (ref) & 1 (ref) & 1 (ref) \\
\hline & Primary education & $0.77(0.41-1.45)$ & $1.43(0.90-2.27)$ & $0.62(0.29-1.30)$ & $1.23(0.75-2.02)$ \\
\hline & Secondary education & $0.84(0.42-1.71)$ & $1.60(0.98-2.61)$ & $0.34(0.12-0.94)$ & $1.35(0.79-2.29)$ \\
\hline & University & $0.87(0.38-2.05)$ & $1.56(0.92-2.65)$ & $0.16(0.03-0.80)$ & $1.22(0.68-2.19)$ \\
\hline No. of births & (by each birth) & 1.25 (1.09-1.42) & & $1.15(0.97-1.36)$ & - \\
\hline Premenopause & (ref.: postmenopause) & $1.21(0.50-2.93)$ & & $0.72(0.19-2.75)$ & - \\
\hline \multirow[t]{3}{*}{ Smoking } & No smoker & 1 (ref) & 1 (ref) & 1 (ref) & 1 (ref) \\
\hline & Former smoker & $1.33(0.69-2.58)$ & $1.67(1.14-2.44)$ & $1.00(0.36-2.80)$ & $1.80(1.16-2.79)$ \\
\hline & Current smoker & $1.89(0.96-3.71)$ & $1.64(1.01-2.64)$ & $1.71(0.63-4.65)$ & $1.85(1.07-3.19)$ \\
\hline \multicolumn{6}{|c|}{ Alcohol consumption at recruitment (g/day) } \\
\hline \multicolumn{2}{|l|}{ Abstainers } & 1 (ref) & 1 (ref) & 1 (ref) & 1 (ref) \\
\hline \multicolumn{2}{|c|}{ 0-39.9 (men), 0-19.9 (women) } & $1.14(0.62-2.08)$ & $0.81(0.52-1.27)$ & $1.19(0.54-2.65)$ & $0.96(0.58-1.60)$ \\
\hline \multicolumn{2}{|c|}{ 40-59.9 (men), 20-39.9 (women) } & $0.64(0.15-2.69)$ & $0.49(0.22-1.09)$ & $1.05(0.16-6.98)$ & $0.52(0.21-1.33)$ \\
\hline \multicolumn{2}{|c|}{$\geq 60$ g/day (men), $\geq 40$ g/day (women) } & NA & $1.01(0.41-2.46)$ & - & $1.38(0.52-3.65)$ \\
\hline \multicolumn{6}{|c|}{ Alcohol when $30-40$ years old } \\
\hline \multicolumn{2}{|l|}{ Abstainers } & 1 (ref) & 1 (ref) & 1 (ref) & 1 (ref) \\
\hline \multicolumn{2}{|c|}{ 0-39.9 (men), 0-19.9 (women) } & $1.00(0.55-1.83)$ & $1.73(1.00-3.00)$ & $1.08(0.49-2.40)$ & $1.40(0.77-2.53)$ \\
\hline \multicolumn{2}{|c|}{ 40-59.9 (men), 20-39.9 (women) } & $2.16(0.75-6.26)$ & $1.44(0.72-2.89)$ & $2.61(0.62-10.9)$ & $1.13(0.52-2.45)$ \\
\hline \multicolumn{2}{|c|}{$\geq 60$ g/day (men), $\geq 40$ g/day (women) } & NA & $1.44(0.74-2.78)$ & - & $1.23(0.60-2.53)$ \\
\hline \multicolumn{6}{|c|}{ Chronic disease involving pain } \\
\hline \multicolumn{2}{|l|}{ Yes (ref: No) } & $1.27(0.78-2.08)$ & $1.23(0.89-1.70)$ & $0.71(0.36-1.36)$ & $1.10(0.76-1.59)$ \\
\hline
\end{tabular}

relationship between NSAID use and current cardiovascular disease or risk factors. Results are shown in Table 6. Men with diabetes mellitus consumed less non-aspirin NSAIDs -and, specifically, less propionates- than men without that condition; no such result could be found for women. Having hypertension, hypercholesterolemia or medical history of acute coronary syndrome (i.e.: acute myocardial infarction or angina), stroke or other circulatory diseases was not associated with lower non-aspirin NSAID consumption in any gender. Using aspirin, however, increased in both women and men suffering any of these cardiovascular risk factors or diseases, with $\mathrm{OR}$ as higher as 15.2 (women with acute coronary syndrome), 13.3 (women having had a stroke) or 11.1 (men with acute coronary syndrome).

\section{Discussion}

According to our results, NSAID consumption differed by gender and age, being aspirin more used by men and older participants and propionates by women and the youngsters. Consumption of any major NSAID group was consistently associated with educational level in men: the higher the educational level, the higher the NSAID usage. Regarding health-related behaviours, current smoker women had ever consumed more 
Table 5 Factors associated with acetate acid derivate consumption: odds ratios and 95\% confidence intervals adjusted for the remaining factors in the table and province of recruitment

\begin{tabular}{|c|c|c|c|c|c|}
\hline \multirow[t]{2}{*}{ Variable } & \multirow[t]{2}{*}{ Category } & \multicolumn{2}{|c|}{ Ever consumption } & \multicolumn{2}{|c|}{ Current consumption } \\
\hline & & Women & Men & Women & Men \\
\hline \multirow[t]{5}{*}{ Age } & $<45$ & $1.12(0.51-2.43)$ & $0.76(0.22-2.59)$ & $0.86(0.26-2.81)$ & $2.88(0.39-21.2)$ \\
\hline & $45-54$ & 1.00 (ref) & 1 (ref) & 1 (ref) & 1 (ref) \\
\hline & $55-64$ & $0.86(0.47-1.55)$ & $0.58(0.28-1.19)$ & $0.73(0.29-1.84)$ & $1.44(0.33-6.27)$ \\
\hline & $65-74$ & $0.66(0.34-1.26)$ & $0.52(0.26-1.06)$ & $0.52(0.18-1.45)$ & $0.96(0.22-4.19)$ \\
\hline & $\geq 75$ & $0.81(0.39-1.68)$ & $0.48(0.22-1.06)$ & $0.73(0.24-2.24)$ & $1.20(0.25-5.62)$ \\
\hline \multirow[t]{4}{*}{ BMl } & $<18.5$ & $0.27(0.03-2.13)$ & $2.62(0.17-40.8)$ & $0.78(0.09-6.66)$ & - \\
\hline & $18.5-24.9$ & 1.00 (ref) & 1 (ref) & 1 (ref) & 1 (ref) \\
\hline & $25-29.9$ & $1.30(0.84-2.00)$ & $1.22(0.76-1.97)$ & $1.67(0.87-3.22)$ & $0.49(0.22-1.06)$ \\
\hline & $\geq 30$ & $1.57(0.97-2.56)$ & $1.32(0.74-2.34)$ & $1.27(0.57-2.83)$ & $0.81(0.34-1.94)$ \\
\hline \multirow[t]{4}{*}{ Education } & Less than primary & 1 (ref) & 1 (ref) & 1 (ref) & 1 (ref) \\
\hline & Primary education & $1.14(0.67-1.96)$ & $1.34(0.70-2.57)$ & $0.69(0.30-1.58)$ & $1.45(0.46-4.57)$ \\
\hline & Secondary education & $0.86(0.46-1.59)$ & $2.27(1.20-4.28)$ & $0.58(0.23-1.50)$ & $1.93(0.63-5.89)$ \\
\hline & University & $1.41(0.73-2.71)$ & $2.15(1.09-4.27)$ & $1.29(0.49-3.35)$ & $2.40(0.73-7.87)$ \\
\hline No. of births & (by each birth) & $0.97(0.85-1.10)$ & & $1.12(0.94-1.33)$ & - \\
\hline \multicolumn{2}{|c|}{ Premenopause (ref.: postmenopause) } & $0.45(0.23-0.86)$ & & $0.58(0.22-1.49)$ & - \\
\hline \multirow[t]{3}{*}{ Smoking } & No smoker & 1 (ref) & 1 (ref) & 1 (ref) & 1 (ref) \\
\hline & Former smoker & $1.20(0.73-1.99)$ & $0.91(0.57-1.44)$ & $0.94(0.42-2.08)$ & $1.66(0.71-3.84)$ \\
\hline & Current smoker & $1.72(1.03-1.99)$ & $0.91(0.51-1.63)$ & $1.33(0.59-2.99)$ & $0.96(0.31-3.02)$ \\
\hline \multicolumn{6}{|c|}{ Alcohol consumption at recruitment (g/day) } \\
\hline \multicolumn{2}{|l|}{ Abstainers } & 1 (ref) & 1 (ref) & 1 (ref) & 1 (ref) \\
\hline \multicolumn{2}{|c|}{ 0-39.9 (men), 0-19.9 (women) } & $0.90(0.55-1.48)$ & $1.14(0.59-2.19)$ & $1.01(0.46-2.21)$ & $0.88(0.27-2.81)$ \\
\hline \multicolumn{2}{|c|}{ 40-59.9 (men), 20-39.9 (women) } & $1.27(0.50-3.24)$ & $1.98(0.83-4.73)$ & $0.85(0.19-3.81)$ & $1.28(0.27-6.08)$ \\
\hline \multicolumn{2}{|c|}{$\geq 60$ g/day (men), $\geq 40$ g/day (women) } & $1.80(0.26-12.3)$ & $1.40(0.46-4.31)$ & $3.43(0.18-64.1)$ & $2.80(0.50-15.6)$ \\
\hline \multicolumn{6}{|c|}{ Alcohol when $30-40$ years old } \\
\hline \multicolumn{2}{|l|}{ Abstainers } & 1 (ref) & 1 (ref) & 1 (ref) & 1 (ref) \\
\hline \multicolumn{2}{|c|}{ 0-39.9 (men), 0-19.9 (women) } & $1.24(0.75-2.04)$ & $0.75(0.39-1.41)$ & $1.35(0.61-2.97)$ & $7.92(0.95-66.0)$ \\
\hline \multicolumn{2}{|c|}{ 40-59.9 (men), 20-39.9 (women) } & $2.23(0.95-5.22)$ & $1.08(0.49-2.37)$ & $4.28(1.27-14.5)$ & $7.98(0.84-76.2)$ \\
\hline \multicolumn{2}{|c|}{$\geq 60 \mathrm{~g} /$ day (men), $\geq 40 \mathrm{~g} /$ day (women) } & $1.35(0.36-5.08)$ & $0.87(0.40-1.89)$ & - & $3.32(0.32-34.6)$ \\
\hline \multicolumn{6}{|c|}{ Chronic disease involving pain } \\
\hline \multicolumn{2}{|c|}{ Yes (ref: No) } & $2.69(1.81-4.00)$ & $3.49(2.33-5.21)$ & $3.23(1.72-6.06)$ & $4.28(2.10-8.71)$ \\
\hline
\end{tabular}

non-aspirin NSAIDs but current smoker men had lower current consumption of non-aspirin NSAIDs than no smoker women and men, respectively. Higher current consumption was found in current heavy ethanol drinkers. People with higher risk of cardiovascular adverse episodes when taking non-aspirin NSAIDs (i.e.: participants with cardiovascular disease history or cardiovascular risk factors) consumed as much non-aspirin NSAID as people without such a high risk, the only exception being men with diabetes mellitus, who halved the non-aspirin NSAID current consumption. Nonetheless, participants with higher cardiovascular risk took aspirin more frequently.

\section{Consumption compared with other countries}

Ibuprofen was by far the more consumed NSAID in our study. Higher use of ibuprofen has also been informed in Germany [17], the US [18] and Denmark [19]. A study on 15 countries, however, reported diclofenac as the most frequently used, followed by ibuprofen [20]. Many articles have reported NSAID trend use [17, 21], which has not been analysed in our study. However, the trend in Spain seemed to be rising until 2009 and slowly decreasing from then on; specifically, ibuprofen reaching its zenith in 2009, while naproxen began to increase in 2012 [22]. Qato et al. [7] informed of increases in NSAID drugs from 2005 to 2011 in the US, with aspirin 
Table 6 Association between cardiovascular disease or risk factors and current NSAID consumption (odds ratios and 95\% confidence intervals adjusted by age, BMl, educational level, smoking, province of recruitment and presence of arthritis, gout or chronic cephalalgia)

\begin{tabular}{|c|c|c|c|}
\hline NSAID & Cardiovascular disease or risk factor & Women & Men \\
\hline \multirow[t]{6}{*}{ Non-aspirin NSAID } & Diabetes mellitus & $1.06(0.70-1.62)$ & $0.59(0.37-0.95)$ \\
\hline & Hypertension & $1.02(0.76-1.38)$ & $0.81(0.57-1.31)$ \\
\hline & Hypercholesterolemia & $1.13(0.86-1.47)$ & $1.05(0.75-1.47)$ \\
\hline & Acute coronary syndrome & $0.64(0.29-1.43)$ & $0.86(0.51-1.44)$ \\
\hline & Stroke & $1.00(0.58-1.13)$ & $1.11(0.51-2.43)$ \\
\hline & Other circulatory diseases & $0.81(0.58-1.13)$ & $0.99(0.63-1.58)$ \\
\hline \multirow[t]{6}{*}{ Propionic derivates } & Diabetes mellitus & $1.28(0.79-2.06)$ & $0.56(0.30-1.04)$ \\
\hline & Hypertension & $0.94(0.66-1.32)$ & $0.81(0.54-1.23)$ \\
\hline & Hypercholesterolemia & $1.23(0.90-1.66)$ & $1.06(0.70-1.59)$ \\
\hline & Acute coronary syndrome & $1.07(0.46-2.52)$ & $0.63(0.31-1.30)$ \\
\hline & Stroke & $0.85(0.32-2.22)$ & $0.45(0.11-1.90)$ \\
\hline & Other circulatory diseases & $0.72(0.49-1.08)$ & $0.71(0.38-1.35)$ \\
\hline \multirow[t]{6}{*}{ Aspirin } & Diabetes mellitus & $4.36(2.39-7.94)$ & $2.44(1.73-3.43)$ \\
\hline & Hypertension & $4.98(2.48-9.98)$ & $2.44(1.73-3.43)$ \\
\hline & Hypercholesterolemia & $2.15(1.22-3.79)$ & $3.62(2.58-5.07)$ \\
\hline & Acute coronary syndrome & $15.2(7.43-31.2)$ & $11.1(7.81-15.9)$ \\
\hline & Stroke & $13.3(6.24-28.3)$ & $2.09(1.19-3.66)$ \\
\hline & Other circulatory diseases & $3.86(2.16-6.90)$ & $3.56(2.51-5.04)$ \\
\hline \multirow[t]{6}{*}{ Acetic acid derivates } & Diabetes mellitus & $0.81(0.33-1.99)$ & $0.95(0.45-2.01)$ \\
\hline & Hypertension & $1.39(0.76-2.52)$ & $1.01(0.54-1.89)$ \\
\hline & Hypercholesterolemia & $1.36(0.78-2.35)$ & $0.84(0.45-1.58)$ \\
\hline & Acute coronary syndrome & - & $0.69(0.26-1.84)$ \\
\hline & Stroke & $1.77(0.51-6.12)$ & $1.75(0.58-5.31)$ \\
\hline & Other circulatory diseases & $1.53(0.83-2.80)$ & $0.97(0.43-2.19)$ \\
\hline
\end{tabular}

use increasing from 30.2 to $40.2 \%$ and other NSAIDs from 10.15 to $13.7 \%$. Differences in definitions and methodology among the studies, however, makes it difficult to compare figures from different countries.

\section{Patterns by age and sex}

Few studies reported NSAID pattern consumption by age. Like ours, Dale et al. [23] informed of an increase in aspirin and a decrease in other NSAIDs with age in Norway; Sarganas et al. [17] also reported that NSAID consumption was lower with higher age in Germany. Clinical guidelines recommend restricting non-aspirin NSAIDs in the older group because people at a higher age have higher risks of NSAID-related adverse episodes, both gastrointestinal haemorrhage and cardiovascular events [1, 3], so our results are in accordance with this. The interpretation of the age-pattern of aspirin is challenging as its consumption would be as an analgesic/anti-inflammatory drug or as anti-aggregant. In our study, aspirin was strongly associated with cardiovascular risk factors, such as hypertension and hypercholesterolemia, but especially with previous cardiovascular diseases, such as acute coronary syndrome and strokes. Aspirin is well-known as a drug able to produce gastrointestinal haemorrhage, especially in aged people, but also for its cardiovascular protective effects when used in low doses. Being in no doubt of its usage for secondary prevention in people already affected by ischemic cardiovascular disease, current US Preventive Service Task Force [24] recommendation for cardiovascular disease primary prevention, however, only supports using aspirin in people aged between 50 and 59, with possible extension on individual basis until 69 years old, but no longer as evidence of the risk/benefit relationship in patients older than 70 was considered insufficient [25]. Women consumed more NSAIDs than men, as previously reported in several articles [17, 23]. In our study, arthritis and chronic cephalalgia were more frequent in women, confirming other studies which suggested that non-malignant chronic diseases causing pain are more prevalent in women [26], eventually leading to more analgesic / anti-inflammatory usage. 


\section{Education level}

Education level could be used as a surrogate for socio-economic level. It was positively associated with NSAID use in our study. These results, however, are in contrary to those found in Germany $[7,17]$ which studied education and house income as different variables with similar findings: a positive association with NSAIDs: the higher the education level or the higher the house income, the more frequent the NSAID consumption is.

\section{Health-related behaviours}

Among currently smokers, we found that women had higher ever non-aspirin NSAIDs consumption and men had lower current non-aspirin NSAIDs current or former smoker men had consumed more aspirin. Current alcohol consumption had a positive but non-significant association with non-aspirin NSAIDs. Dale et al. found a positive association of NSAID consumption with current smoking and a negative one with alcohol [23], while both alcohol and tobacco use were positively associated with NSAIDs in Sweden about 20 years previously [27]. As suggested in Dale et al., these differences may echo cultural and social changes throughout that period [23].

\section{Differences in current and ever NSAID consumption}

Most patterns of consumption were quite similar for current and ever consumers. Non-aspirin NSAIDs were more consumed by more educated men and women, while aspirin were more consumed by older people; these patterns were more marked in current than in ever consumers, which is probably indicating recent trends. To interpret differences among current and ever consumption, however, is speculative as data on both consumptions are prone to different biases; in this regard, we focused more on similarities than on differences as similar patterns could be considered some kind of confirmatory results.

\section{Public health implications}

Recommendations for prescribing NSAIDs have been developed in guidelines [3, 4] regarding their risk profile on gastrointestinal haemorrhage and cardiovascular episodes. They agree in considering naproxen as being less prone to cardiovascular episodes than ibuprofen, thus they recommended using naproxen if a non-aspirin NSAID is needed in patients with high cardiovascular risk, especially if they are taking aspirin for cardio-protection. However, the FDA (Food and Drug Administration), in a safety announcement for advising on cardiovascular risks associated with NSAIDs, stated there is not enough evidence to determine that a specific non-aspirin NSAID has higher or lower cardiovascular risk than any other [5]. Therefore, the relevance of higher use of ibuprofen than naproxen -as we reported- is unclear. On the other hand, older people, which are at high risk of cardiovascular events or gastrointestinal haemorrhage, tend to use less non-aspirin NSAIDs and more aspirin than people at low risk. Finally, although the higher consumption of aspirin by people with cardiovascular diseases or risk factors would be related with its usage as secondary prevention, the fact that the same high cardiovascular risk people did not report lower non-aspirin NSAID consumption brings up a point of concern because these NSAIDs could put them at higher cardiovascular risk.

\section{Strenghs and limitations}

Our study has some limitations. Firstly, information on NSAID use was obtained in a face-to-face interview, so it would be affected by recall bias and social desirability bias as well; moreover, no information was recorded on prescriptions in order to validate whether the information provided in the interview accurately represented actual NSAID consumption. On the other hand, some subjects could consider that drugs obtained over-the-counter -as occurs with many NSAID, especially aspirin- are not really medicines, leading to underreport their usage. Secondly, the sample is formed by the control group in a case-control study on several types of cancer; although participants in the study were selected at random, some cancer cases had to be excluded. This fact could bias the results towards lower NSAID consumption than the general population. Thirdly, although our subjects were selected at random, we cannot exclude that people agreeing to participate could be self-selected because of their health behaviours or interests, which could limit the generalization of our results. Fourth, our data did not allow us to distinguish whether aspirin is being taken as painkiller or for cardiovascular prevention purposes.

By the other hand, our study has also some strengths within his study. Firstly, we have a large sample from 12 different Spanish provinces, which makes our results more reliable. Secondly, the vast amount of information gathered as part of a case-control study on cancer allows us the analysis of determinants of NSAID consumption. It is noteworthy that participants were not aware of any hypothesis regarding NSAID usage when they were interviewed, which makes unlikely the presence of differential biases associated with the reported informations.

\section{Conclusion}

Summarizing, we found that propionates are the most consumed group of NSAIDs in Spain. Consumption of non-aspirin NSAIDs was associated with demographic groups with lower gastrointestinal and cardiovascular risk; however, participants at high cardiovascular risk had no lower non-aspirin NSAID consumption, which points out some concerns on the current NSAID consumption or prescription in Spain. 


\section{Additional files}

Additional file 1: NSAID-group ever use by age and sex [\%, (95\% CI)].
(PDF $47 \mathrm{~kb}$ )

Additional file 2: NSAID-group current use by age and sex [\%, (95\% Cl)]. (PDF $43 \mathrm{~kb}$ )

Additional file 3: NSAID ever group use by province of recruitment [\%, (95\% Cl)]. (PDF $21 \mathrm{~kb})$

Additional file 4: Consumption of specific non-aspirin NSAIDs. Only active principles with reported anytime-consumption over $1 \%$ are included. Data indicate percentage and 95\% confidence interval. (PDF 36 kb)

\section{Abbreviations}

ATC: Therapeutic Classification of Drugs; CEIC: Comité Ético de Investigación Clínica; Cl: \% confidence intervals; FDA: Food and Drug Administration; FFQ: Food Frequency Questionnaire; GI: Gastrointestinal; MCC: Multicasecontrol; NSAIDs: Non-steroidal anti-inflammatory drugs; OR: Odds ratios

\section{Funding}

This work was partially funded by the "Accion Transversal del Cancer", approved on the Spanish Ministry Council on the 11th October 2007; The Instituto de Salud Carlos III-FEDER [PI08/1770, PI08/0533, PI08/1359, PI09/ 00773-Cantabria, PI09/01286-León, PI09/01903-Valencia, PI09/02078-Huelva, PI09/01662-Granada, PI11/01403, PI11/01889-FEDER, PI11/00226, PI11/01810, PI11/02213, PI12/00488, PI12/00265, PI12/01270, PI12/00715, PI12/00150 PI14/01219, PI14/0613, PI15/00069, PI15/00914, PI15/01032]; The Fundación Marqués de Valdecilla [API 10/09]; The ICGC International Cancer Genome Consortium CLL (The ICGC CLL-Genome Project is funded by Spanish Ministerio de Economía y Competitividad (MINECO) through the Instituto de Salud Carlos III (ISCIII) and Red Temática de Investigación del Cáncer (RTICC) del ISCIII (RD12/0036/0036)); The Junta de Castilla y León [LE22A10-2]; The Consejería de Salud of the Junta de Andalucía [2009-S0143]; The Conselleria de Sanitat of the Generalitat Valenciana [AP_061/10]; The Recercaixa [2010ACUP 00310]; The Regional Government of the Basque Country; The Consejería de Sanidad de la Región de Murcia; The European Commission [grants FOODCT-2006-036224-HIWATE]; The Spanish Association Against Cancer (AECC) Scientific Foundation; The Catalan Government DURSI [grant 2014SGR647]; The Fundación Caja de Ahorros de Asturias; and the University of Oviedo. The funding body had no role in the design of the study and collection, analysis, and interpretation of data and in writing the manuscript.

\section{Availability of data and materials}

The informed consent obtained from the study participants prevents the full data from being made publicly available. The anonymized dataset necessary to replicate this study's findings will be available upon request to the corresponding author.

\section{Authors' contributions}

Study conception and design: $J$ and IGA. Statistical analysis: TDS, MP, CPC, JAM, IG-A, JL. Coordination of substudy sites, recruitment, acquisition of data read and revised the manuscript: GCV, TFV, PA, RC, RP, JMA, VDB, BPG, JE, YB, GFT, ISB, RM, JMH, AT, AB, NA, MP, MK. Drafting of the manuscript: IGA and $J$. Contributions to the final version of the manuscript were made by all authors. All authors read and approved the final manuscript.

\section{Ethics approval and consent to participate}

Participants who agreed to partake in the study signed an informed consent before the face-to-face interview, and the protocol of MCC-Spain was approved by the local Ethics Committees of participating institutions (Comité Ético de Investigación Clínica (CEIC) del Instituto Municipal de Asistencia Sanitaria de Barcelona; CEIC del Hospital Universitario de Bellvitge; CEIC de Navarra; CEIC del Hospital Universitario La Paz; CEIC del Hospital Universitario Ramón y Cajal; CEIC de Cantabria; CEIC de Gipuzkoa; CEIC de Granada; CEIC de Murcia; CEIC de Valencia; CEIC de Girona; Comité de Ética de la Investigación de la Provincia de Huelva; CEIC de León; Comité Ético de Investigación del Principado de Asturias), in conformity to the principles of the Declaration of Helsinki. The database was registered in the Spanish Agency for Data Protection (no. 2102672171).

\section{Consent for publication}

Not applicable.

\section{Competing interests}

The authors declare that they have no competing interests.

\section{Publisher's Note}

Springer Nature remains neutral with regard to jurisdictional claims in published maps and institutional affiliations.

\section{Author details}

${ }^{1}$ CIBER Epidemiología y Salud Pública, Madrid, Spain. ${ }^{2}$ Facultad de Medicina, Universidad de Cantabria - IDIVAL, Avda Herrera Oria s/n, 39011 Santander, Spain. ${ }^{3}$ Department of Obstetrics and Gynecology, Nuevo Belén University Hospital, Madrid, Spain. ${ }^{4}$ Environmental and Cancer Epidemiology Unit, National Centre for Epidemiology, Carlos III Institute of Health (Instituto de Salud Carlos III-ISCIII), Avda. Monforte de Lemos, 5, 28029 Madrid, Spain. ${ }^{5}$ Institute of Health Research "Puerta de Hierro", IDIPHIM, Madrid, Spain. ${ }^{6}$ ISGlobal, Centre for Research in Environmental Epidemiology (CREAL), Barcelona, Spain. ${ }^{7}$ Universitat Pompeu Fabra (UPF), Barcelona, Spain. ${ }^{8} \mathrm{IMIM}$ (Hospital del Mar Medical Research Institute), Barcelona, Spain. ${ }^{9}$ Institute of Biomedicine (IBIOMED), University of León, León, Spain. ${ }^{10}$ Public Health Division of Gipuzkoa, BioDonostia Research Institute, San Sebastian, Spain. ${ }^{11}$ Department of Statistics and O.R., INAMAT, Public University of Navarre, Pamplona, Spain. ${ }^{12}$ Unit of Infections and Cancer, Cancer Epidemiology Research Programme, IDIBELL, Catalan Institute of Oncology, Barcelona, Spain. ${ }^{13}$ University of Oviedo, Oviedo, Spain. ${ }^{14}$ Universidad de Granada, Granada, Spain. ${ }^{15}$ Centro de Investigación en Recursos Naturales, Salud, y Medio Ambiente. (RENSMA), Universidad de Huelva, Huelva, Spain. ${ }^{16}$ Dirección General de Salud Pública, Fundación para el fomento de la investigación sanitaria y biomédica de la Comunidad Valenciana, FISABIO-Salud Pública, Valencia, Spain. ${ }^{17}$ Unitat d'Epidemiologia i Registre de Càncer de Girona (UERCG), Pla Director d'Oncologia, Institut Català d'Oncologia, Institut d'Investigaciò Biomèdica de Girona (IdIBGi), Universitat de Girona, Girona, Spain. ${ }^{18}$ Department of Epidemiology, Murcia Regional Health Council, IMIB-Arrixaca, Murcia, Spain. ${ }^{19}$ Navarra Public Health Institute, Pamplona, Spain. ${ }^{20}$ Navarra Institute for Health Research (IdiSNA), Pamplona, Spain. ${ }^{21}$ Osakidetza-Basque Health Service, BioDonostia Research Institute, Donostia, Spain. ${ }^{22}$ Epidemiology Section, Public Health Division, Department of Health of Madrid, Madrid, Spain.

Received: 30 November 2017 Accepted: 4 September 2018 Published online: 21 September 2018

\section{References}

1. Scarpignato $\mathrm{C}$, Hunt $\mathrm{RH}$. Nonsteroidal antiinflammatory drug-related injury to the gastrointestinal tract: clinical picture, pathogenesis, and prevention. Gastroenterol Clin N Am. 2010:39:433-64.

2. Fanelli A, Ghisi D, Aprile PL, Lapi F. Cardiovascular and cerebrovascular risk with nonsteroidal anti-inflammatory drugs and cyclooxygenase 2 inhibitors: latest evidence and clinical implications. Ther Adv Drug Saf. 2017:8:173-82.

3. Chan FKL, Abraham NS, Scheiman JM, Laine L. First international working party on gastrointestinal and cardiovascular effects of nonsteroidal antiinflammatory drugs and anti-platelet agents. Management of patients on nonsteroidal anti-inflammatory drugs: a clinical practice recommendation from the First International Working Party on Gastrointestinal and Cardiovascular Effects of Nonsteroidal Anti-inflammatory Drugs and Antiplatelet Agents. Am J Gastroenterol. 2008;103:2908-18.

4. Scarpignato $C$, Lanas A, Blandizzi C, Lems WF, Hermann M, Hunt RH, et al. Safe prescribing of non-steroidal anti-inflammatory drugs in patients with osteoarthritis--an expert consensus addressing benefits as well as gastrointestinal and cardiovascular risks. BMC Med. 2015;13:55.

5. FDA. Drug Safety and Availability - FDA Drug Safety Communication: FDA strengthens warning that non-aspirin nonsteroidal anti-inflammatory drugs (NSAIDs) can cause heart attacks or strokes. 2015. https://www.fda.gov/ Drugs/DrugSafety/ucm451800.htm. Accessed 8 Sept 2017.

6. Agencia Española de Medicamentos y Productos Sanitarios Medicamentos de Uso Humano - Observatorio del Uso de Medicamentos de la AEMPS - Informes publicados. https://www. aemps.gob.es/medicamentosUsoHumano/observatorio/informes.htm. Accessed 23 July 2018. 
7. Qato DM, Wilder J, Schumm LP, Gillet V, Alexander GC. Changes in prescription and over-the-counter medication and dietary supplement use among older adults in the United States, 2005 vs 2011. JAMA Intern Med. 2016;176:473-82.

8. Davis JS, Lee HY, Kim J, Advani SM, Peng H-L, Banfield E, et al. Use of nonsteroidal anti-inflammatory drugs in US adults: changes over time and by demographic. Open Heart. 2017;4:e000550.

9. Kaufman DW, Kelly JP, Wiholm BE, Laszlo A, Sheehan JE, Koff RS, et al. The risk of acute major upper gastrointestinal bleeding among users of aspirin and ibuprofen at various levels of alcohol consumption. Am J Gastroenterol. 1999:94:3189-96.

10. Chi T-Y, Zhu H-M, Zhang M. Risk factors associated with nonsteroidal antiinflammatory drugs (NSAIDs)-induced gastrointestinal bleeding resulting on people over 60 years old in Beijing. Medicine (Baltimore). 2018;97:e0665.

11. Sostres C, Carrera-Lasfuentes P, Lanas A. Non-steroidal anti-inflammatory drug related upper gastrointestinal bleeding: types of drug use and patient profiles in real clinical practice. Curr Med Res Opin. 2017;33:1815-20.

12. Castaño-Vinyals G, Aragonés N, Pérez-Gómez B, Martín V, Llorca J, Moreno V, et al. Population-based multicase-control study in common tumors in Spain (MCC-Spain): rationale and study design. Gac Sanit. 2015;29:308-15.

13. MCC Spain - Multi-Caso Control Spain. http://www.mcespain.org/. Accessed 18 Jul 2018

14. García-Closas R, García-Closas M, Kogevinas M, Malats N, Silverman D, Serra $C$, et al. Food, nutrient and heterocyclic amine intake and the risk of bladder cancer. Eur J Cancer Oxf Engl 1990. 2007;43:1731-40.

15. 03_Cuestionario-alimentario_09Nov09.pdf. http://www.mccspain.org/wpcontent/uploads/2016/04/03_Cuestionario-alimentario_09Nov09.pdf. Accessed 18 Jul 2018.

16. Rehm Jürgen, Room Robin, Monteiro Maristela, Gmel Gerhard, Graham Kathryn, Rehn Nina, et al. Alcohol use. In: Comparative quantification of health risks : global and regional burden of disease attributable to selected major risk factors. 2004. http://www.who.int/publications/cra/chapters/ volume1/0000i-xxiv.pdf. Accessed 8 Sep 2017.

17. Sarganas G, Buttery AK, Zhuang W, Wolf I-K, Grams D, Rosario AS, et al. Prevalence, trends, patterns and associations of analgesic use in Germany. BMC Pharmacol Toxicol. 2015;16:28.

18. Wilcox CM, Cryer B, Triadafilopoulos G. Patterns of use and public perception of over-the-counter pain relievers: focus on nonsteroidal antiinflammatory drugs. J Rheumatol. 2005;32:2218-24.

19. Schjerning Olsen A-M, Fosbøl EL, Lindhardsen J, Folke F, Charlot M, Selmer C, et al. Duration of treatment with nonsteroidal antiinflammatory drugs and impact on risk of death and recurrent myocardial infarction in patients with prior myocardial infarction: a nationwide cohort study. Circulation. 2011;123:2226-35.

20. McGettigan P, Henry D. Use of non-steroidal anti-inflammatory drugs that elevate cardiovascular risk: an examination of sales and essential medicines lists in low-, middle-, and high-income countries. PLoS Med. 2013;10:e1001388.

21. Mijatović V, Calasan J, Horvat O, Sabo A, Tomić Z, Radulović V. Consumption of non-steroidal anti-inflammatory drugs in Serbia: a comparison with Croatia and Denmark during 2005-2008. Eur J Clin Pharmacol. 2011;67:203-7.

22. AEMPS. Utilización de medicamentos antiinflamatorios no esteroides (AINE) en España durante el periodo 2000-2012. 2014. https://www. aemps.gob.es/medicamentosUsoHumano/observatorio/docs/AINE.pdf. Accessed 8 Sep 2017.

23. Dale O, Borchgrevink PC, Fredheim OMS, Mahic M, Romundstad P, Skurtveit S. Prevalence of use of non-prescription analgesics in the Norwegian HUNT3 population: impact of gender, age, exercise and prescription of opioids. BMC Public Health. 2015;15:461.

24. USPSTF. US Preventive Services Task Force. https://www. uspreventiveservicestaskforce.org/. Accessed 8 Sept 2017

25. Dehmer SP, Maciosek MV, Flottemesch TJ, LaFrance AB, Whitlock EP. Aspirin for the primary prevention of cardiovascular disease and colorectal Cancer: a decision analysis for the U.S. preventive services task force. Ann Intern Med. 2016;164:777.

26. Fillingim RB. Sex, gender, and pain: women and men really are different. Curr Rev Pain. 2000;4:24-30.

27. Antonov K, Isacson D. Use of analgesics in Sweden--the importance of sociodemographic factors, physical fitness, health and health-related factors, and working conditions. Soc Sci Med 1982. 1996;42:1473-81.

\section{Ready to submit your research? Choose BMC and benefit from:}

- fast, convenient online submission

- thorough peer review by experienced researchers in your field

- rapid publication on acceptance

- support for research data, including large and complex data types

- gold Open Access which fosters wider collaboration and increased citations

- maximum visibility for your research: over $100 \mathrm{M}$ website views per year

At $\mathrm{BMC}$, research is always in progress.

Learn more biomedcentral.com/submissions 